\title{
The importance of accurate imaging diagnosis of a vascular anomaly prior to the surgical procedure
}

\author{
Maria Popescu, Gabriel Dragan, Alina Oprescu \\ Department of Pediatric Surgery, "Victor Gomoiu" Clinical Hospital for Children, Bucharest, Romania
}

\begin{abstract}
The diagnosis of vascular tumors and malformations is usually achieved with clinical history and physical examination. The imaging of these patients has been sufficiently refined to answer pertinent questions when trying to make an accurate differential diagnosis in order to establish an informed treatment plan. The imaging modalities at hand include ultrasound, doppler sonography, magnetic resonance imaging and contrast material enhanced magnetic resonance angiography.

We present two cases of different vascular anomalies that were managed according to the clinical aspect, natural history and imaging performed.

Moreover, with better understanding of the imaging features, the radiologist has become a key player in the diagnosis and management plan of the vascular anomalies. This is crucial in avoiding misdiagnosis and improper treatment.
\end{abstract}

Keywords: vascular anomalies, magnetic resonance imaging, pediatric patients, misdiagnosis

\begin{abstract}
Abbreviations
ISSVA - International Society for the Study of Vascular Anomalies

MRI - magnetic resonance

MRA - magnetic resonance angiography
\end{abstract}

\section{INTRODUCTION}

The large area of the vascular anomalies is currently divided by the International Society for the Study of Vascular Anomalies (ISSVA) into vascular tumors and vascular malformations. Due to the lack of knowledge regarding the clinical and histopathologic aspect of vascular anomalies and also of their origin, the terminology that most doctors use is often confusing (1). Different types of vascular anomalies need different treatment methods making the overlapping of names an impediment to implement the best therapeutic management that is needed for the patient. ISSVA developed a system that correlates imaging terminology with the personal history of the patient, clinical course, histologic diagnosis and the adequate treatment (2).
The imaging methods largely available nowadays that are used both to confirm the diagnosis of different types of vascular anomalies, as well to delineate the lesions further, are the magnetic resonance (MRI) and ultrasound, along with magnetic resonance angiography (MRA) $(3,4)$.

The most important part of imaging vascular anomalies includes characterizing the lesion and establishing the anatomic extent of the lesion. For the vascular malformations knowing the tissues and the adjacent vital structures that are involved is vital to planning surgery or other imaging guided procedures $(5,6)$. Only the clinical examination does not give enough relevant information for a perfect management plan.

We report two cases of vascular anomalies that received extended imaging diagnosis before sur- 
gery and the management plan was established according to MRA findings and the intra-operatory aspect of the lesion. The outcomes were very different.

\section{CASES PRESENTATION}

\section{Case 1}

\section{Presenting concerns}

We present the case of a 3-year-old girl that was admitted to our surgical department to investigate a vascular anomaly on the left cheek that received treatment in another pediatric surgery department. We were able to obtain the personal history of the patient from the mother that described the presence of the lesion since birth and the fact that the pathology evolved slowly and proportionate with the growth of the child. The diagnosis at one month was that of an infantile hemangioma and received medical treatment for one year with Propranolol, that did not affect in any way the evolvement of the vascular anomaly. After the age of one year and a half, the patient was injected with Bleomycin in the attempt to perform sclerotherapy of the lesion, with no result after 5 attempts, 2 months apart from each other. The only imaging investigations that she had were doppler ultrasounds by different radiologists.

\section{Clinical findings and diagnostic focus}

The initial clinical examination described a subcutaneous mass of $3 / 3 \mathrm{~cm}$ on the left cheek, poorly delimited, elastic under palpation, increased temperature of the epidermis and non-painful. The color of the skin above the lesion is bluish, and it modifies according to the exterior conditions, becoming more reddish when the temperature increases, under direct sunlight or when the child is crying (Fig. 1).

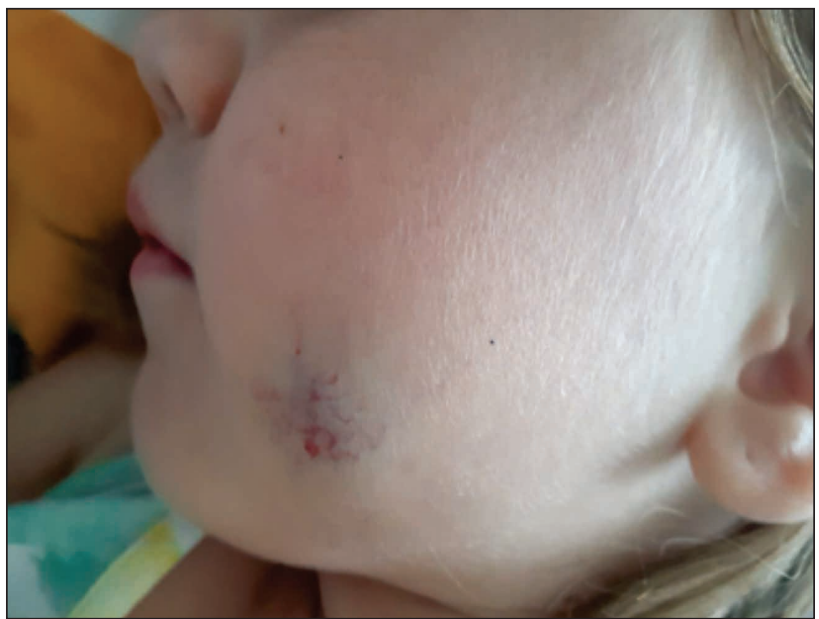

FIGURE 1. Clinical aspect of the first case
We revised the treatment methods that were applied, the personal history and the clinical aspect and we raised the suspicion of a misdiagnosed vascular malformation instead of an infantile hemangioma.

We performed a doppler ultrasound that assessed the vascularity of the anomaly and the types of vessels feeding the lesion. It was challenging to determine the exact extend of the malformation with the ultrasound, its range being limited, so we took the next step and submitted the patient to an MRA that described the presence of a probable arterial vascular malformation with arterial feeding vessels form the left internal and external carotid and draining veins from the left jugular. Important posterior contact with the facial artery and vein (Fig. 2 and 3).

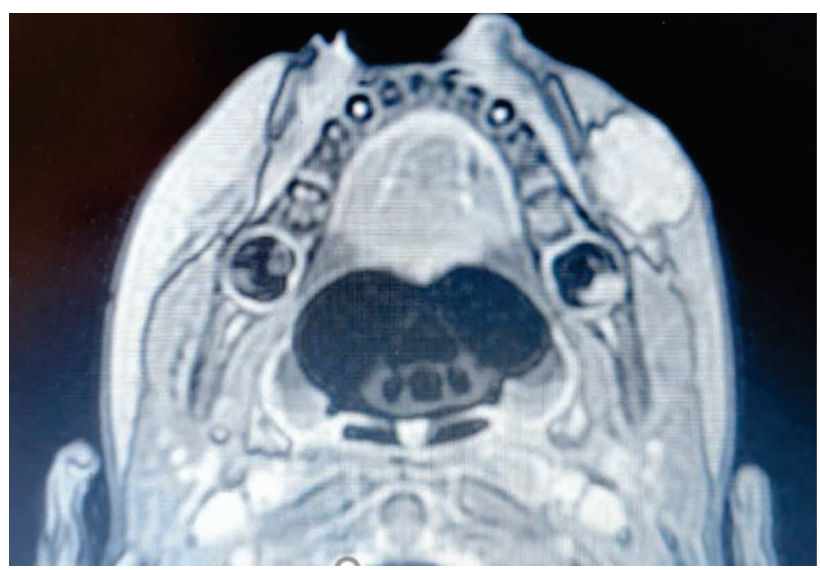

FIGURE 2. MRI aspect of the first case

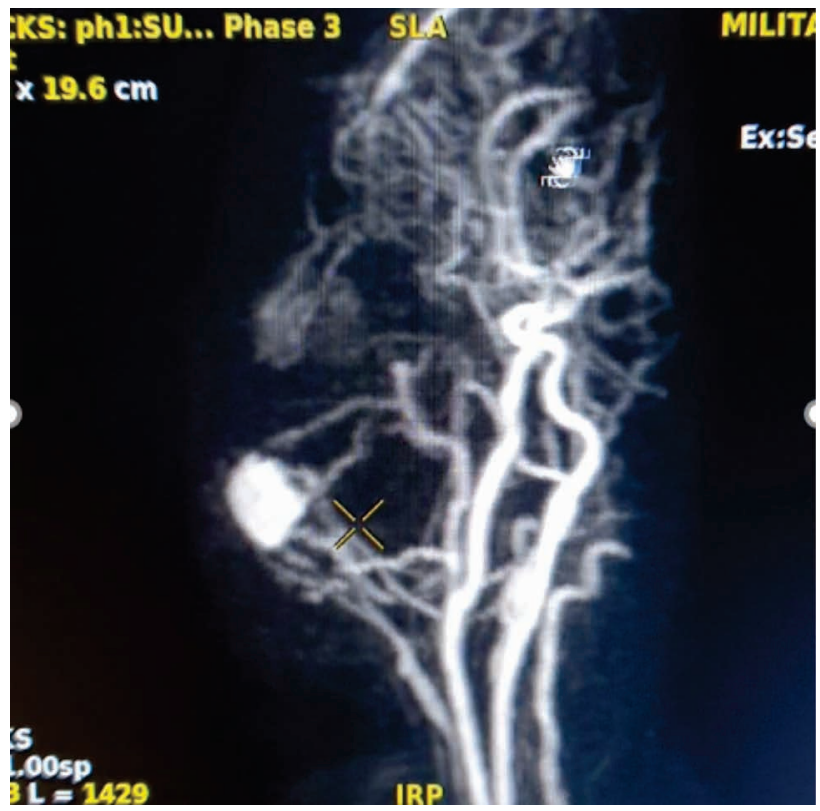

FIGURE 3. MRA aspect of the first case

\section{Therapeutic focus and assessment}

Following further discussions with the parents, we decided to perform the surgical removal of the 
vascular anomaly. The procedure took place under general anesthesia. We excised the entire vascular anomaly and the tegument above it, in order to limit the potential of a relapse. We dissected and ligated the feeding arteries and the draining veins. The facial artery and vein were not included in the anomaly and were easily dissected. No drain was necessary (Fig. 4 and 5).

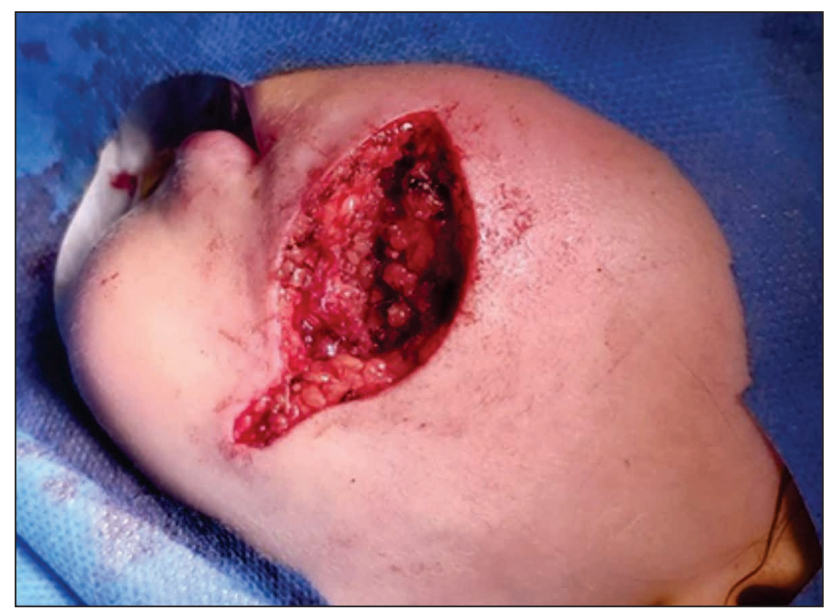

FIGURE 4. Intra-operatory aspect of the first case

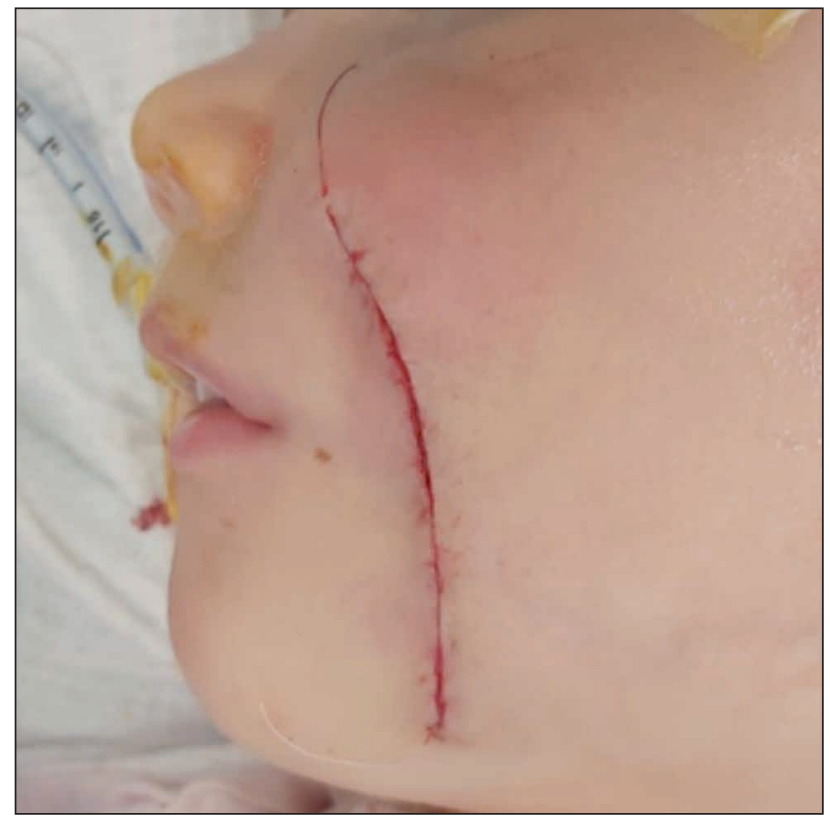

FIGURE 5. Intra-operatory aspect of the first case

\section{Follow-up and monitoring}

The post operatory evolution was without incidences. The patient received only one dosage of antibiotic for protection and was discharged 24 hours post-surgery (Fig. 6).

The histopathologic result revealed the diagnosis of a non-involuting hemangioma $(\mathrm{NICH})$, a type of vascular tumor that can mimic a vascular malformation.

Follow ups for the first year revealed no relapse of the vascular anomaly.

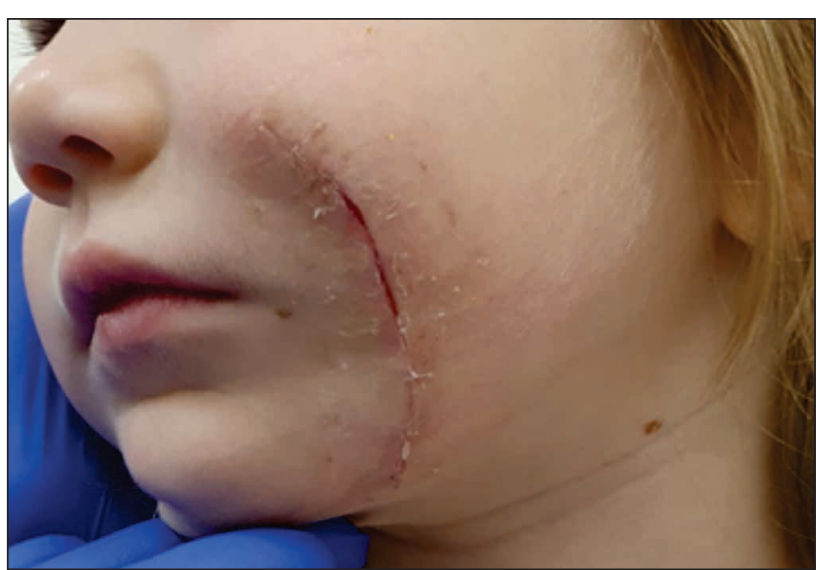

FIGURE 6. One month follow-up for the first case, scar aspect

\section{Case 2}

\section{Presenting concerns}

The second case, is that of a 6 months old girl that was admitted to our surgical department to investigate and treat the presence of a frontal mass. We obtained the personal history of the patient from the parents, that described the presence of the mass since birth and the fact that it evolved slowly and proportionate with the growth of the child.

\section{Clinical findings and diagnostic focus}

The clinical examination of the patient revealed the presence of a $2 / 2 \mathrm{~cm}$ frontal subcutaneous mass, well delimited from the tissue around, elastic under palpation and not painful. The skin above the mass had a bluish discoloration with underlying fullness and the temperature was not elevated. The aspect of the mass did not change under exterior conditions or with the distress of the child (Fig. 7).

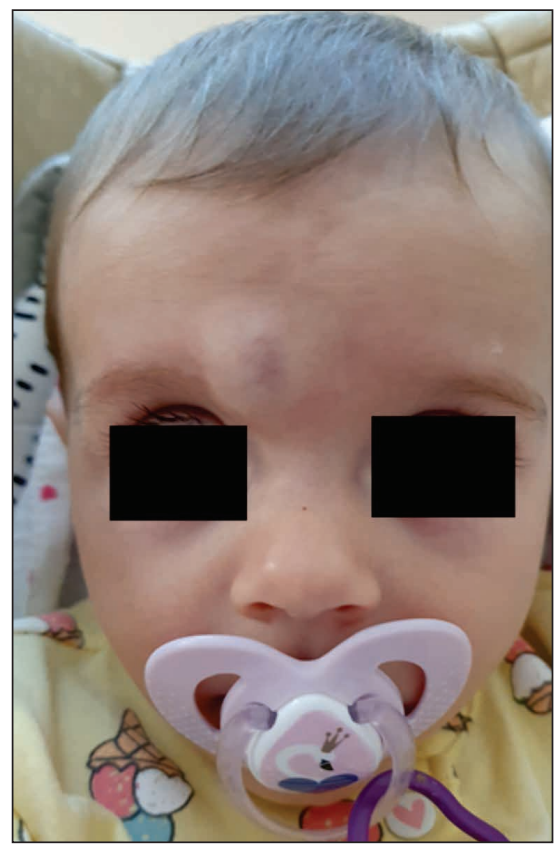

FIGURE 7. Clinical aspect of the second case 
We decided to perform a doppler ultrasound that assessed the low flow vascularity of the mass and an MRA that raised the suspicion of a venous malformation with draining veins from the internal jugular. The mass included the supraorbital and the supratrochlear veins and arteries (Fig. 8).

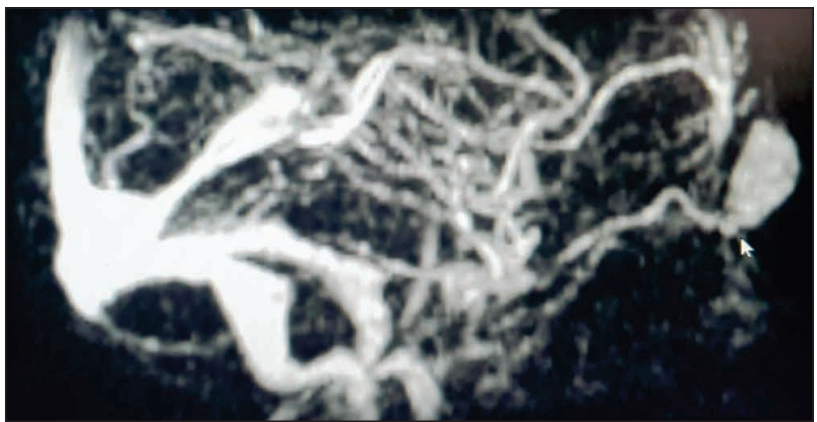

FIGURE 8. MRA aspect of the second case

\section{Therapeutic focus and assessment}

Following further discussion with the parents we decided to perform the surgical excision of the mass. Intra-operatory the tumor seems to be limited to the muscular layer of the region, not affecting the skin level or the profound tissues, with a minimum vascularization. We decided not to excise the tegument above the vascular anomaly as it appeared not affected. We inserted a small drain in order to avoid a post-operatory local hematoma.

\section{Follow up and monitoring}

The drain was removed 24 hours after surgery and the patient was discharged in 48 hours. She received only one prophylactic antibiotic dose during surgery (Fig. 9).

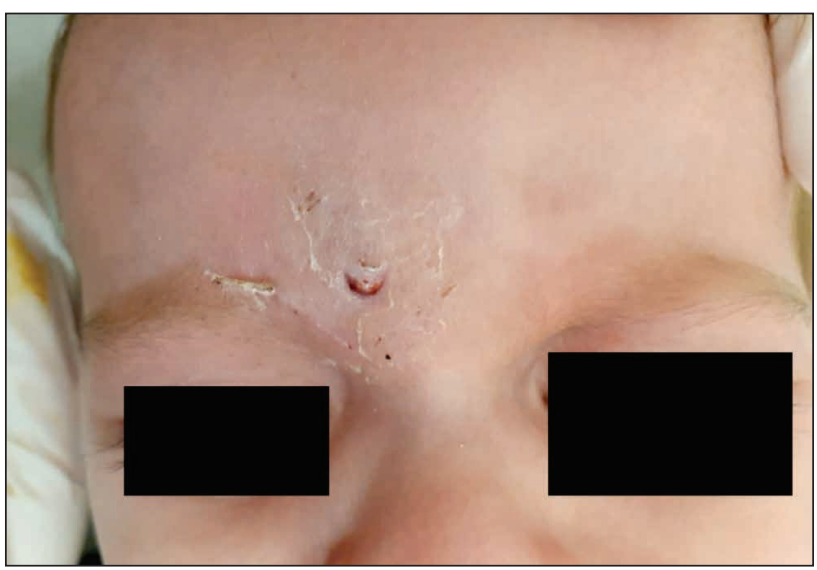

FIGURE 9. Follow-up pictures of the second case, scar aspect

The histopathologic result was that of a venous malformation.

At the one month follow up we observed the presence of a small bluish mass above the surgical scar that became bigger in the following months and has now reached the initial preoperatory dimensions. The recurrence is still to be investigated and further treatment is needed.

\section{DISCUSSIONS}

Vascular tumors and malformations can cause significant problems in both children and even adults. For many reasons physicians frequently confuse these lesions and the terms used are often interchangeably and inappropriately, rendering the therapeutic management of the pathology confusing.

The care of children with vascular anomalies requires the expertise of multiple subspecialities such as pediatric surgery, pediatric cardiology, pediatric radiology, pediatric dermatology and other pediatric specialties according to the affected area of the pathology. The combined skills and knowledge of these subspecialists helps establishing the most appropriate management plan for each case. The large choice of treatments that can be used for different vascular anomalies (medical treatment, sclerotherapy, laser therapy, surgical excision) makes the preoperatory diagnosis an essential part of the therapeutic plan.

Many of the patients that we see in our clinic have previously seen several physicians and received controversary or very few information regarding the vascular anomaly pathology. Some patients have undergone multiple therapeutic procedures usually resulting in little improvement or even a worsening of their symptoms. Radical surgery has been recommended to many of these cases and controversially many other children who might benefit from other therapeutic methods have been treated with watchful waiting worsening the outcome of the pathology in the end. The principal confusion appears to be the differential diagnosis between an infantile hemangioma that can eventually resolve by itself and a vascular malformation that needs more imaging and an adequate treatment plan.

When confronting with such confusing information the personal history of the patient, the clinical aspect and the imaging are essential for the patient and doctor. The right asked questions can lead to an easy diagnosis and an effective management plan. We present in figure 10 the differential diagnosis between an infantile hemangioma and a vascular malformation.

On physical examination only, determining whether the subcutaneous tissue, the underlying muscular layer, or both are involved is difficult. 


\begin{tabular}{|ll|}
\hline \multicolumn{1}{|c|}{ HEMANGIOMAS } & VASCULAR MALFORMATIONS \\
- Not present at birth & - Always present at birth \\
- Proliferation period $\rightarrow$ & - They grow together with \\
maturation phase $\rightarrow$ & the child - no proliferation \\
stabilization & phase \\
- Slow and complete & - No spontaneous regression \\
regression & - Normal no of endothelial \\
- Endothelial cells $\uparrow$ no & cells \\
\hline
\end{tabular}

FIGURE 10. Differential diagnosis of hemangiomas and vascular malformations

The primary goals of imaging a vascular anomaly includes the characterization of the lesion and a potential differential diagnosis (1). When the physical examination and history of the patient are highly suggestive for a vascular malformation, the most important characterization is whether the lesion is a high or low flow malformation. Most information needed is available nowadays by performing an MRI, MR angiography, venography and lymphangiography $(2,3)$.

Ultrasound has been advocated as useful in examining soft tissue masses that are suggestive for vascular anomalies and the use of the doppler sonography can orient a diagnosis. We also use ultrasound for potential therapeutic procedures such as sclerotherapy of a vascular lesion $(3,7)$.

Referring to the two cases that we presented we have to emphasize on the importance of the preoperatory imaging that were performed. In the first case the patient received multiple and long-term treatments that only postponed the surgical excision that was necessary from the beginning. The MRA was essential for the appropriate surgical planning of the operation as the mass involved important vascular findings such as the facial artery and vein. Even if the final histopathologic diagnosis was not of an arterial vascular malformation, but a NICH, as shown in the literature, the optimal choice of treatment was the surgical one. One of Conflict of interest: none declared Financial support: none declared

\section{REFERENCES}

1. Lowe LH, Marchant TC, Rivard DC, Scherbel AJ. Vascular malformations: classification and terminology the radiologist needs to know. Semin Roentgenol. 2012;47(2):106-117.

2. Kollipara R, Dinneen L, Rentas KE, Saettele MR, Patel SA, Rivard DC, Lowe LH. Current classification and terminology of pediatric vascular anomalies. AJR Am J Roentgenol. 2013 Nov;201(5):1124-35.

3. Paltiel HJ, Burrows PE, Kozakewich HP, Zurakowski D, Mulliken JB. Soft-tissue vascular anomalies: utility of US for diagnosis. Radiology. 2000 Mar;214(3):747-54

4. Dubois J, Alison M. Vascular anomalies: what a radiologist needs to know. Pediatr Radiol. 2010 Jun;40(6):895-905.

5. Jarrett DY, Ali M, Chaudry G. Imaging of vascular anomalies. Dermatol Clin. 2013;31(2):251-66.

6. El-Merhi F, Garg D, Cura M, Ghaith O. Peripheral vascular tumors and vascular malformations: imaging (magnetic resonance imaging the most accepted reasons for performing a surgical procedure on a vascular anomaly is the non-involuting feature of the pathology with or without other treatments. The imaging is important in quantifying the dimensions of the lesions, ultrasound being more useful than MRI for frequent follow-ups $(7,8)$.

For the second case that we presented the intra-opera operatory aspect was not suggestive of a vascular malformation and a personal momentary decision was taken not to excise the underlying skin tissue in order to try to minimize the important scaring of the facial area. The recurrence of the pathology and the histopathologic result demonstrated that that the preoperatory imaging was right once again and that the correct attitude is to establish the surgical plan in conformity to these findings. The literature data shows a $50 \%$ chance of recurrence in case of a subtotal excision of a vascular malformation (9). If the tegument above the anomaly is affected the correct procedure is to excise it, disregarding the esthetic part of the surgical process and emphasizing on the absolute need of total ablation of the lesion (10).

\section{CONCLUSIONS}

Vascular anomalies are endothelial lesions that can present with serious medical problems both for children and adults. Knowing the differential diagnosis between an infantile hemangioma and a vascular malformation is essential for the management plan of each case. Moreover, the multidisciplinary team approach of the vascular anomalies has led to better patient care and outcomes. Radiology has become an essential component of the team approach as it provides the most accurate diagnosis and is essential for the preoperatory management of complicated cases.

and conventional angiography), pathologic correlation and treatment options. Int J Cardiovasc Imaging. 2013;29(2):379-93.

7. Marler JJ, Mulliken JB. Vascular anomalies: classification, diagnosis, and natural history. Facial Plast Surg Clin North Am. 2001;9(4):495-504.

8. Haggstrom AN, Drolet BA, Baselga E, et al; Hemangioma Investigator Group. Prospective study of infantile hemangiomas: demographic, prenatal, and perinatal characteristics. J Pediatr. 2007;150(3):291-94.

9. Restrepo R. Multimodality imaging of vascular anomalies. Pediatr Radiol. 2013;43(Suppl (Suppl 1):S141-S154.

10. Kollipara R, Odhav A, Rentas KE, Rivard DC, Lowe LH, Dinneen L. Vascular anomalies in pediatric patients: updated classification, imaging, and therapy. Radiol Clin North Am. 2013;51(4):659-672. 